\title{
ALMOST ORTHOGONAL SERIES
}

\section{RICHARD BELLMAN}

1. Almost orthogonal series. Let us consider an infinite sequence $\left\{\phi_{n}(x)\right\}, n=1,2, \cdots$, of complex-valued functions of the real variable $x$, of class $L^{2}(a, b)$, normalized so that $\int_{a}^{b}\left|\phi_{n}(x)\right|^{2} d x=1$ for all $n$. Assume further that the sequence satisfies the following condition

$$
\sum_{m, n}\left|a_{m n}\right|^{2}<\infty,
$$

where $a_{m n}=\int_{a}^{b} \phi_{m} \bar{\phi}_{n} d x(m \neq n ; n, m=1,2, \cdots), a_{m n}=0, m=n$.

We wish to show that under the above conditions we have a Bessel inequality and an analogue of the Riesz-Fisher theorem.

TheOREM 1 (Bessel's INEqUality). Under the above conditions, let $f(x)$ be a real-valued function belonging to $L^{2}(a, b)$, and $b_{n}=\int_{a}^{b} \bar{\phi}_{n} d x$, then

We have

$$
\sum_{1}^{\infty}\left|b_{k}\right|^{2} \leqq \int_{a}^{b}|f|^{2} d x\left[1+\left(\sum_{m, n}\left|a_{m n}\right|^{2}\right)^{1 / 2}\right] .
$$

$$
\sum_{1}^{n}\left|b_{k}\right|^{2}=\int_{a}^{b} f\left[\sum_{1}^{n} b_{k} \bar{\phi}_{k}\right] d x .
$$

Using Schwartz's inequality, this becomes

$$
\begin{aligned}
\sum_{1}^{n}\left|b_{k}\right|^{2} \leqq & {\left[\int_{a}^{b}|f|^{2} d x\right]^{1 / 2}\left[\int_{a}^{b}\left[\sum_{1}^{n} b_{k} \phi_{k}\right]\left[\sum_{1}^{n} b_{k} \bar{\phi}_{k}\right] d x\right]^{1 / 2} } \\
\leqq & {\left[\int_{a}^{b}|f|^{2} d x\right]^{1 / 2}\left[\sum_{1}^{n}\left|b_{k}\right|^{2}+\sum_{1,1, k \neq l}^{n, n} b_{k} b_{l} a_{k l}\right]^{1 / 2} } \\
\leqq & {\left[\int_{a}^{b}|f|^{2} d x\right]^{1 / 2}\left[\sum_{1}^{n}\left|b_{k}\right|^{2}\right.} \\
& \left.+\left\{\sum_{1,1}^{n, n}\left|b_{k}\right|^{2}\left|b_{l}\right|^{2}\right\}^{1 / 2}\left\{\sum_{1,1}^{n, n}\left|a_{k l}\right|^{2}\right\}^{1 / 2}\right]^{1 / 2} \\
\leqq & {\left[\int_{a}^{b}|f|^{2} d x\right]^{1 / 2}\left[\sum_{1}^{n}\left|b_{k}\right|^{2}\right]^{1 / 2} } \\
& \cdot\left[1+\left(\sum_{m, n}\left|a_{k l}\right|^{2}\right)^{1 / 2}\right]^{1 / 2} .
\end{aligned}
$$

Received by the editors, July 28, 1943, and, in revised form, November 26, 1943. 
Hence, simplifying,

$$
\sum_{1}^{n}\left|b_{k}\right|^{2} \leqq\left(\int_{a}^{b}|f|^{2} d x\right)\left[1+\left(\sum_{m, n}\left|a_{k l}\right|^{2}\right)^{1 / 2}\right],
$$

and since this is true for all $n$, the result is obtained.

Theorem 2 (ANAlogue of Riesz-Fisher). Under the above conditions, if $\sum_{1}^{\infty}\left|b_{n}\right|^{2}<\infty$, there exists a function $f(x) \subset L^{2}(a, b)$ such that

$$
\sum_{1}^{\infty}\left|b_{k}-\int_{a}^{b} f \bar{\phi}_{k} d x\right|^{2} \leqq\left(\sum_{1}^{\infty}\left|b_{k}\right|^{2}\right)\left(\sum_{k, l}\left|a_{k l}\right|^{2}\right)
$$

and therefore $\lim _{n \rightarrow \infty}\left(b_{n}-\int_{a}^{b} \overline{\phi_{n}} d x\right)=0$.

Let $s_{n}=\sum_{1}^{n} b_{k} \phi_{k}$, then

$$
\begin{aligned}
\int_{a}^{b}\left|s_{m}-s_{n}\right|^{2} d x & \\
& =\int_{a}^{b}\left[\sum_{n+1}^{m} b_{k} \phi_{k}\right]\left[\sum_{n+1}^{m} b_{k} \bar{\phi}_{k}\right] d x \\
& =\sum_{n+1}^{m}\left|b_{k}\right|^{2}+\sum_{n+1 \leqq k, l \leqq m} b_{k} b_{l} a_{k l} \\
& \leqq \sum_{n+1}^{m}\left|b_{k}\right|^{2}+\left(\sum_{n+1}^{m}\left|b_{k}\right|^{2}\left|b_{l}\right|^{2}\right)^{1 / 2}\left(\sum_{m, n}\left|a_{m n}\right|^{2}\right)^{1 / 2} \\
& \leqq \sum_{n+1}^{m}\left|b_{k}\right|^{2}\left(1+\left(\sum_{m, n}\left|a_{m n}\right|^{2}\right)^{1 / 2}\right)
\end{aligned}
$$

(using Schwartz's inequality). Hence, since $\sum b_{n}^{2}<\infty, s_{n}$ converges in mean to a function $f(x) \subset L^{2}(a, b)$, that is,

$$
\lim _{n \rightarrow \infty} \int_{a}^{b}\left|f(x)-s_{n}(x)\right|^{2} d x=0 .
$$

We have, $k<n$,

$$
\begin{aligned}
b_{k}-\int_{a}^{b} f \bar{\phi}_{k} d x & =b_{k}-\int_{a}^{b} s_{n} \bar{\phi}_{k} d x+\int_{a}^{b}\left(s_{n}-f\right) \bar{\phi}_{k} d x \\
\int_{a}^{b}\left|s_{n}-f\right|\left|\phi_{k}\right| d x & \leqq\left(\int_{a}^{b}\left|f-s_{n}\right|^{2} d x\right)^{1 / 2}\left(\int_{a}^{b}\left|\phi_{k}\right|^{2} d x\right)^{1 / 2} \\
& =\left(\int_{a}^{b}\left|f-s_{n}\right|^{2} d x\right)^{1 / 2}
\end{aligned}
$$




$$
\begin{aligned}
b_{k}-\int_{a}^{b} s_{n} \bar{\phi}_{k} d x & =\sum_{j=1}^{n} b_{j} a_{k j} \\
\left|b_{k}-\int_{a}^{b} s_{n} \bar{\phi}_{k} d x\right| & \leqq \sum_{1}^{n}\left|b_{j} a_{k j}\right| \\
& \leqq\left(\sum_{1}^{n}\left|b_{j}\right|^{2}\right)^{1 / 2}\left(\sum_{j=1}^{n}\left|a_{k j}\right|^{2}\right)^{1 / 2}
\end{aligned}
$$

Hence,

$$
\begin{aligned}
\left|b_{k}-\int_{a}^{b} f \bar{\phi}_{k} d x\right| \leqq & \left(\sum_{1}^{n}\left|b_{j}\right|^{2}\right)^{1 / 2}\left(\sum_{j=1}^{n}\left|a_{k j}\right|^{2}\right)^{1 / 2} \\
& +\left(\int_{a}^{b}\left|f-s_{n}\right|^{2} d x\right)^{1 / 2} .
\end{aligned}
$$

Let $n \rightarrow \infty$, this becomes

$$
\left|b_{k}-\int_{a}^{b} f \bar{\phi}_{k} d x\right| \leqq\left(\sum_{1}^{\infty}\left|b_{j}\right|^{2}\right)^{1 / 2}\left(\sum_{j=1}^{\infty}\left|a_{k j}\right|^{2}\right)^{1 / 2} .
$$

Squaring both sides, and summing over $k$,

$$
\sum_{k}\left|b_{k}-\int_{a}^{b} f \bar{\phi}_{k} d x\right|^{2} \leqq\left(\sum_{1}^{\infty}\left|b_{j}\right|^{2}\right)\left(\sum_{k, j}\left|a_{k j}\right|^{2}\right),
$$

which is the result in question.

2. An "almost" moment problem. Let us consider the sequence of functions $\left\{e^{i \lambda_{n} t} /(b-a)^{1 / 2}\right\}$, the $\lambda_{n}$ being real and distinct, over a finite interval $(a, b)$. Then we have the following theorem.

Theorem 3. If $\sum_{k \neq l} 1 /\left(\lambda_{k}-\lambda_{l}\right)^{2}<\infty$, and $\sum_{n}\left|b_{n}\right|^{2}<\infty$, there exists a function $f(t) \subset L^{2}(a, b)$, such that

$$
\sum_{n}\left|b_{n}-\frac{1}{(b-a)^{1 / 2}} \int_{a}^{b} f(t) e^{-i \lambda_{n} t} d t\right|^{2}<\infty .
$$

We have

$$
\left|\int_{a}^{b} e^{i \lambda_{l} t} e^{-i \lambda_{k} t} d t\right| \leqq \frac{2}{\left|\lambda_{k}-\lambda_{l}\right|}, \quad k \neq l .
$$

Therefore, in view of the hypothesis, this is a corollary of Theorem 2.

Princeton University 